\title{
A EFETIVIDADE DO PLANO DIRETOR DE REGIONALIZAÇÃO DO SUS NO RIO GRANDE DO NORTE
}

\section{the effectiveness of the SUS regionalization director plan in Rio Grande do Norte}

\author{
Aldo Dantas ${ }^{1}$ \\ Luciana da Costa Feitosa ${ }^{2}$ \\ aaaa
}

\begin{abstract}
Resumo
O artigo analisa a efetividade do Plano Diretor de Regionalização (PDR) do Sistema Único de Saúde (SUS) no Rio Grande do Norte a partir dos fluxos funcionais instituídos e sua relação com a dinâmica dos lugares. Os principais aspectos considerados foram as materialidades (condições locais de ordem técnica e sua configuração territorial) e seus componentes organizacionais (leis e normas) vistos em contextos regionais. A hipótese levantada é a de que as geografias são importantes elementos a serem considerados na formulação e execução de políticas públicas. Estas, por seu caráter centrado nos indivíduos, tendem a desconsiderar as singularidades dos lugares, que são mais que uma extensão geométrica do espaço. Sendo assim, a partir das mediações empíricas entre o que está proposto nas normas e a dinâmica territorial do Sistema Único de Saúde, conclui-se que há um descompasso entre a política de regionalização estabelecida pela Secretaria Estadual de Saúde do Rio Grande do Norte e a realidade da assistência à saúde que se dá nos lugares.
\end{abstract}

Palavras-chave: Território, Sistema Único de Saúde, Regionalização, Normas.

\begin{abstract}
This article examines the effectiveness of the Regionalization Plan (RDP) of the Unified Health System (in Portuguese - SUS) in Rio Grande do Norte State based on functional flows introduced and its relation to the dynamics of locations. The main aspects considered were the materiality (local technical conditions and its territorial configuration) and its organizational components (laws and regulations) seen in regional contexts. The hypothesis is that geographies are important elements to be considered in the formulation and implementation of public policies. Such policies, due to their individual centered-character, tend to disregard the uniqueness of the places that are more than an extension of the geometric space. Thus, from the empirical mediations between what is proposed in standards and territorial dynamics of the Health System, it is concluded that there is a mismatch between the regionalization policy established by the State Health Secretariat of Rio Grande do Norte and the reality of health care that occurs in places.
\end{abstract}

Key words: Planning, Health System, Regionalization, Standards.

\section{Resumen}

El artículo examina la eficacia del Plan de Regionalización (RDP) del Sistema Único de Salud (SUS), en Rio Grande do Norte, a partir de flujos funcionales introducidos y su relación con la dinámica de los lugares. Los principales aspectos considerados fueron la materialidad (condiciones técnicas locales y su configuración territorial) y sus componentes organizativos (leyes y reglamentos) que se observan en el contexto regional. La hipótesis es que las geografías son elementos importantes a tener en cuenta en la formulación e implementación de políticas públicas, las cuales, por su carácter centrado en las personas, no tienen en cuenta la singularidad de los lugares que son más que una extensión geométrica del espacio. Así, desde las mediaciones empíricas entre lo que se propone en los estándares y dinámicas territoriales del sistema de salud, se concluye que existe un desajuste entre la política de regionalización establecida por la Secretaría de Salud del Estado de Rio Grande do Norte y la realidad de atención de la salud que se produce en algunos lugares.

Palabras-clave: Territorio, Sistema de salud, regionalización, estándar.

(1) Prof. Dr. da Pós-Graduação em Geografia da Universidade Federal do Rio Grande do Norte - Campus Universitário Lagoa Nova, CEP: 59072-970 - Natal (RN), Brasil. Tel: (+55 84)91936288 - aldodantas@ufrnet.br

(2) Doutoranda do Programa de Pós-Graduação em Geografia da Faculdade de Ciência e Tecnologia da UNESP/Presidente Prudente - Rua Roberto Simonsen, 305, CEP: 19060-900, Presidente Prudente (SP), Brasil. lcostafeitosa@gmail.com

\section{aaAaa}




\section{INTRODUÇÃO}

A regionalização do Sistema Único de Saúde (SUS), estabelecida no artigo 198 da Constituição Federal e regulamentada pelo Decreto $n^{\circ} 7.508$, de 28 de junho de 2011, é a principal estratégia da política do Ministério da Saúde para garantir resolutividade às demandas do sistema em seus diversos níveis de complexidade. O Plano Diretor de Regionalização (PDR) surge como o principal documento que orienta a conformação das regiões de saúde nos estados da federação, o que faz da regionalização uma ferramenta de planejamento a serviço do Estado imprescindível para organizar no território a distribuição das unidades e a acessibilidade da população à rede de serviços do SUS.

No Rio Grande do Norte, a partir do Plano Diretor de Regionalização implantado no ano de 2008 (visualizado no mapa abaixo), foram instituídas oito regiões de saúde sob este modelo que buscam dar maior resolutividade às demandas do sistema. Sendo assim, o objetivo da nossa pesquisa foi analisar a efetividade do Plano Diretor de Regionalização do SUS no estado do Rio Grande do Norte a partir dos fluxos funcionais instituídos e sua relação com a dinâmica dos lugares.

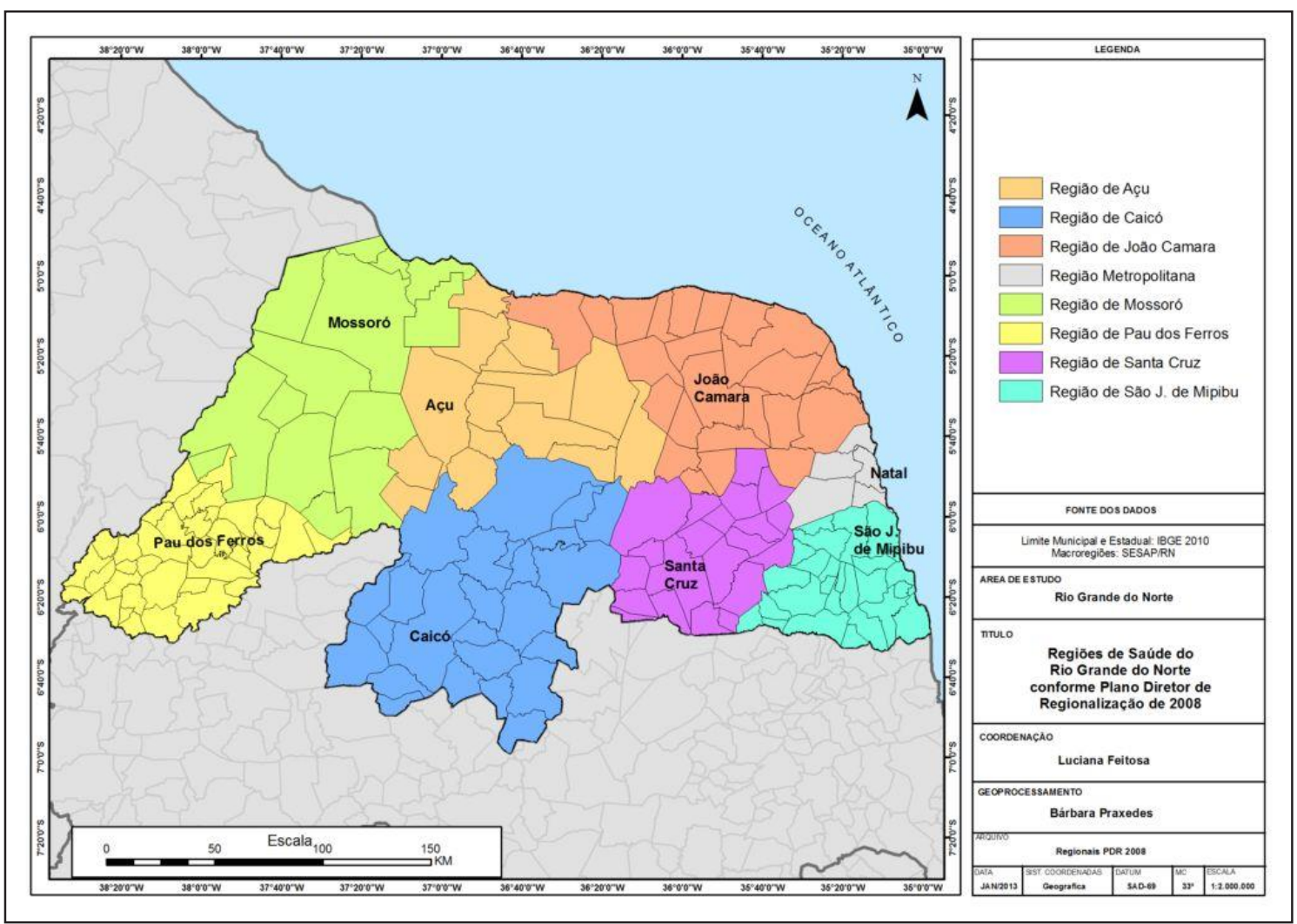

Figura 1 - Localização das Regiões de Saúde do Rio Grande do Norte

\section{MATERIALIDADE, UMA CONDIÇÃO Ȧ AÇÃO}

Para a análise em foco consideramos as materialidades "esse componente imprescindível do espaço geográfico, que é, ao mesmo tempo, uma condição para a ação; uma estrutura de controle, um limite à ação; um convite à ação" (SANTOS, [1996] 2008, p. 321), como a principal variável de estruturação das regiões de saúde no território, pois "nada fazemos hoje que não seja a partir dos objetos que nos cercam” (SANTOS, [1996] 2008, p. 321).

Partimos da ideia de que "as regiões são subdivisões do espaço: do espaço total, do espaço nacional e mesmo do espaço local; são espaços de conveniência, lugares funcionais do todo, um produto social" (SANTOS, 1994, p. 1). Portanto, carregam em sua gênese a coerência entre os lugares que a compõem em virtude das condições locais de ordem técnica (equipamentos, infraestrutura, acessibilidade) e organizacional (leis locais, impostos, relações trabalhistas). 
Os processos que configuram a estrutura organizacional do Sistema Único de Saúde no Rio Grande do Norte não se explicam por si e em si, mas fazem parte de uma teia de relações que envolvem diversos níveis escalares. Para GUIMARÃES (2009, p. 31),

considerar o município isolado de seu contexto regional tem gerado dificuldades por parte das instâncias centrais para formular políticas públicas mais globais. Como as unidades da federação devem obedecer, necessariamente, a divisão jurídico-administrativa do país, esta condição impõe um limite que é, não raras vezes, artificial. Afinal, os processos de adoecer e morrer se assemelham ou se diferenciam independente dos cidadãos viverem em um município ou em outro, assim como as respostas que os serviços de saúde oferecem a cada uma das situações de saúde exigem fluxos e ações que podem extrapolar fronteiras até mesmo entre estados.

Se o SUS deve ser único no território nacional, assegurando os mesmos princípios e diretrizes a toda a população, ele não se realiza da mesma forma em todos os lugares, devido às diferentes heranças e heterogeneidades territoriais. A localização geográfica dos equipamentos tem efeito expressivo na acessibilidade e utilização dos serviços de saúde, comprometendo os ideais de universalidade e equidade no atendimento.

Portanto, sabendo que o Decreto ${ }^{\circ} 7.508$ regulamenta a regionalização como a principal diretriz de organização dos serviços de saúde no território nacional e que a Portaria $n^{\circ} 2.479$ estabelece as Redes de Atenção à Saúde como estratégia para aperfeiçoar o funcionamento do SUS, pudemos analisar a viabilidade dessas normas a partir da estrutura física de cada uma das regiões de saúde instituídas pelo PDR, uma vez que sua principal atribuição é garantir a oferta necessária de serviços e equipamentos de saúde à população que compõe o referido recorte.

Quisemos mostrar a capacidade instalada e os níveis de assistência à saúde que o SUS viabiliza no estado do Rio Grande do Norte. Para isso, fizemos uma análise da distribuição populacional do estado nas regiões de saúde e resgatamos a estrutura das RAS (Redes de Atenção a Saúde) nos seus componentes "Rede de Atenção Básica", "Rede de Atenção às Urgências" e "Rede de Atenção Ambulatorial e Hospitalar" com a finalidade de descobrirmos em que medida essas linhas de cuidado se conectam e são resolutivas.

O Ministério da Saúde define a RAS como "arranjos organizativos de ações e serviços de saúde, de diferentes densidades tecnológicas, que integradas por meio de sistemas de apoio técnico, logístico e de gestão, buscam garantir a integralidade do cuidado". (BRASIL, 2010, p. 89). É uma proposta que visa estabelecer conexões entre os diferentes tipos de atenção à saúde, desde o nível mais básico até o nível mais especializado.

A organização da RAS depende da definição das regiões de saúde e do rol de ações e serviços que serão estabelecidos em cada região. Entre os seus atributos, os principais para a operacionalização da gestão em redes são: 1) população e territórios definidos com amplo conhecimento de suas necessidades; 2) extensa gama de estabelecimentos de saúde e 3) Atenção Primária em Saúde (APS) estruturada como primeiro nível de atenção e porta de entrada do sistema.

Adotamos a Atenção Básica como rede estrutural para a organização e funcionamento do SUS em função de ser uma das estratégias do Ministério da Saúde para reverter o quadro de dependência dos municípios sem ou com poucos estabelecimentos de Média e Alta complexidade aos de maior porte. Além disso, a universalização e consolidação da rede de atenção básica através da Estratégia Saúde da Família é obrigatória a todos os municípios do Brasil desde 1998, uma vez que a eficácia no atendimento neste primeiro nível de atenção resolve entre $80 \%$ e $90 \%$ dos problemas de saúde da população.

Do ponto de vista estrutural o Rio Grande do Norte apresenta cobertura satisfatória da rede de atenção básica, pois dos 167 municípios do estado, 147 apresentam cobertura na Estratégia Saúde da Família igual ou superior a 70\% de sua população. Entretanto, desde 2006 não houve continuidade sistemática do processo, pois boa parte dos municípios do estado parou de cadastrar ou não 
informou cadastramentos de pessoas na ESF, conforme dados obtidos no Sistema de Informação da Atenção Básica (SIAB) para o ano de 2012. Além disso, conforme dados obtidos no Cadastro Nacional dos Estabelecimentos de Saúde no ano de 2012, dos 167 municípios do estado do Rio Grande do Norte 60 não possuem equipes completas nas unidades de saúde da família, o que fere o principio organizativo da rede de atenção básica instituído na Política Nacional de Atenção Básica.

Embora seja o nível de atenção mais capilarizado do território e o que tem o dever de chegar até o domicílio do cidadão, a Rede de Atenção Básica norte-rio-grandense não se completa em sua estrutura organizativa conforme emanam os preceitos da legislação do SUS, pois ao não garantir cobertura populacional a todos os municípios do estado e, além disso, ao não instituir um processo de trabalho com todos os profissionais que compõem a Estratégia Saúde da Família, a "Rede de Atenção Básica" não se faz resolutiva, comprometendo os pontos de conexão entre as redes.

O mesmo acontece no componente "Rede de Atenção às Urgências", que tem no Serviço Móvel de Atendimento às Urgências (SAMU-192) o seu principal componente. O SAMU 192 é um serviço que objetiva chegar precocemente à vítima após ter ocorrido um agravo à sua saúde, sendo necessário garantir atendimento e/ou transporte adequado para um serviço de saúde devidamente hierarquizado e integrado ao SUS.

Até o ano de 2009 o estado do Rio Grande do Norte contava com apenas 03 bases de atendimento do SAMU, com as Centrais de Regulação Médica das Urgências em Natal, (SAMU-Natal), em Mossoró, (SAMU-Mossoró) e no município de Macaíba, (SAMU-Metropolitano). Apenas este último era um Serviço regional que atendia a 08 municípios da região metropolitana de Natal (Ceará Mirim, Extremoz, São Gonçalo do Amarante, Macaíba, Parnamirim, São José de Mipibú, Nísia Floresta e Monte Alegre), que estão divididos em 03 regiões de saúde do estado (Região de Saúde de João Câmara, Região de Saúde Metropolitana e Região de Saúde de São José do Mipibú).

Apesar do projeto de regionalização do SAMU-192 que foi instituído ainda no mesmo ano pela equipe técnica da SESAP/RN, até o ano de 2012 somente a Região de Saúde de João Câmara, Região de Saúde Metropolitana, Região de Saúde de São José do Mipibu e Região de Saúde de Mossoró dispunham de bases em suas jurisdições administrativas, deixando todo o resto do estado com um vazio assistencial no componente Serviço de Atendimento Móvel às Urgências.

A falta de resolutividade das regiões do PDR do Rio Grande do Norte abrange todos os níveis do SUS, chegando inclusive no componente hospitalar, a estrutura de nível mais complexa e hierarquizada do sistema de saúde. Em função de ainda não haver uma regulação específica para este tipo de componente considerando-se a sua função na organização das RAS, tramita nos gabinetes do Ministério da Saúde a implementação da "Política Nacional de Atenção Hospitalar", que tem como objetivo geral estabelecer as diretrizes para a reorganização da Atenção Hospitalar no SUS.

Segundo a Consulta Pública ${ }^{\circ} 19$, de $1^{\circ}$ de novembro de 2012, que vigorou até janeiro de 2013 para opiniões e sugestões acerca das regras que serão estabelecidas na nova Política ministerial, considerou-se que, para serem funcionais, "os hospitais devem ter densidade tecnológica, estrutura física, processos organizativos e profissionais adequados ao seu perfil assistencial e contar com, no mínimo, 50 (cinqüenta) leitos" (BRASIL, 2012, grifos nosso).

Sendo assim, analisamos a capacidade de leitos em cada um dos 23 hospitais estaduais de gestão da SESAP/RN, mais o Hospital Universitário Onofre Lopes (com gestão dupla: federal e estadual) para sabermos até que ponto tais estruturas responderiam às recomendações do Ministério da Saúde caso a Política Nacional de Atenção Hospitalar já estivesse em vigor. Portanto, considerando-se os 23 hospitais estaduais do estado do Rio Grande do Norte, 10 possuem menos de 50 leitos em suas capacidades instaladas, comprometendo a organização do sistema de saúde.

As estruturas materiais condicionam o comportamento dos agentes no território sendo, portanto, imprescindíveis para a análise e formulação de políticas territoriais. Sendo assim, na busca por apreendermos a real dinâmica entre os lugares na busca por atendimento de saúde do SUS no estado cruzamos as informações de prestações de atendimento intermunicipal pactuadas entre os gestores 
de saúde por meio da PPI Ambulatorial e PPI Hospitalar para o ano de 2012 e os dados obtidos no DATASUS (através da ferramenta Tabwin), no período de 2011 (entre janeiro e dezembro).

A partir daí, pudemos sobrepor ao desenho das regiões de saúde o fluxo de deslocamento pactuado com o fluxo de deslocamento real, no intuito de verificarmos se a pactuação que ocorre no nível da gestão é coerente com os deslocamentos do cidadão no território. Para essa análise nos valemos de alguns componentes de prestações de serviços que são estruturantes na política do Ministério da Saúde e que, portanto, devem ser encaradas com maior acuidade pela gestão estadual. Tais componentes foram: encaminhamentos para Parto, mamografias, cirurgias da mama, consultas para ginecologista e obstetrícia, cirurgia obstétrica, cirurgia cardiovascular, encaminhamentos para ortopedia e cirurgia de politraumatismo.

Levando-se em consideração apenas o componente "Parto" chegou-se aos seguintes resultados (que podem ser observados nos mapas e na explicação abaixo):

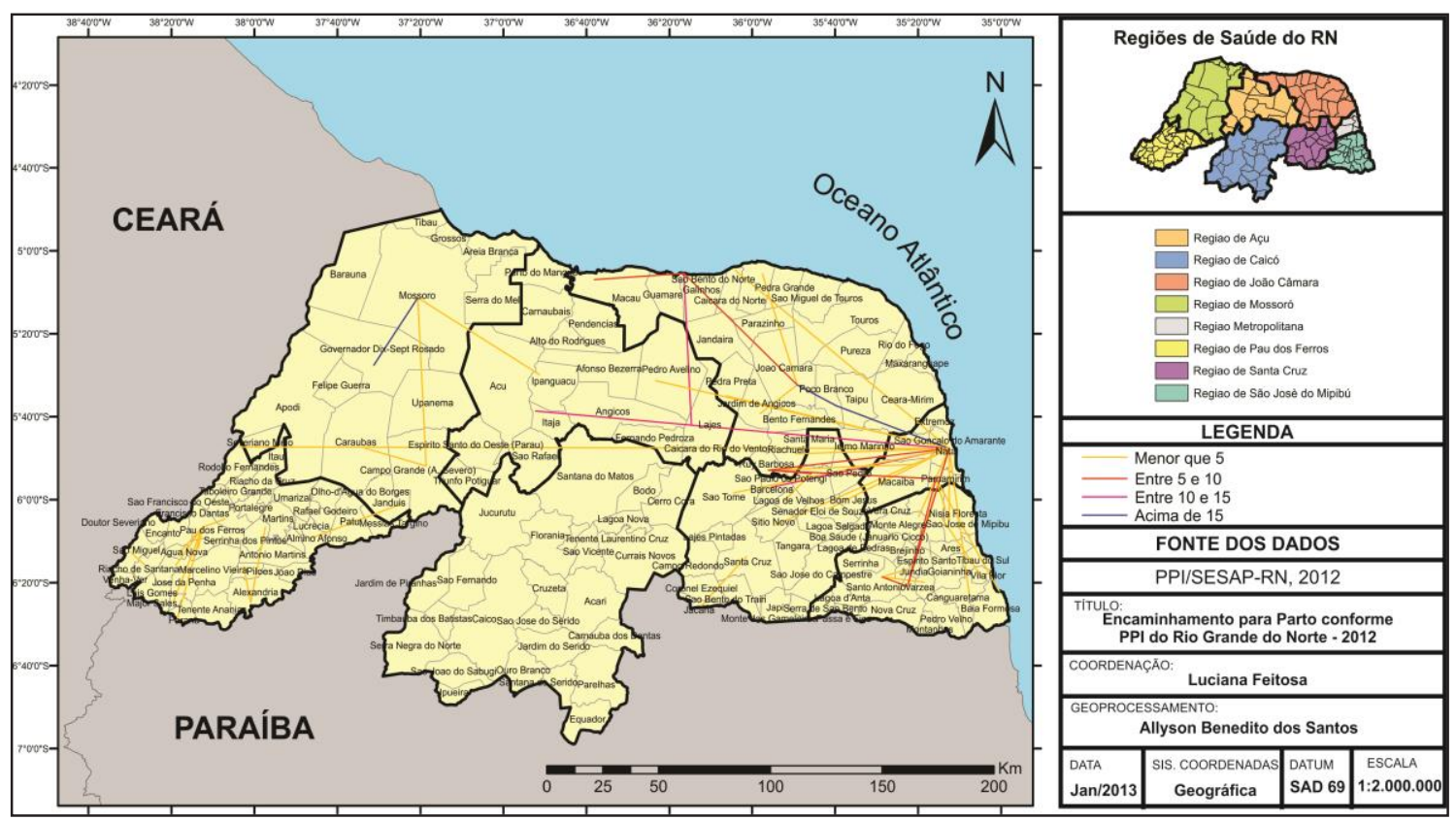

Figura 2 - Encaminhamento para parto
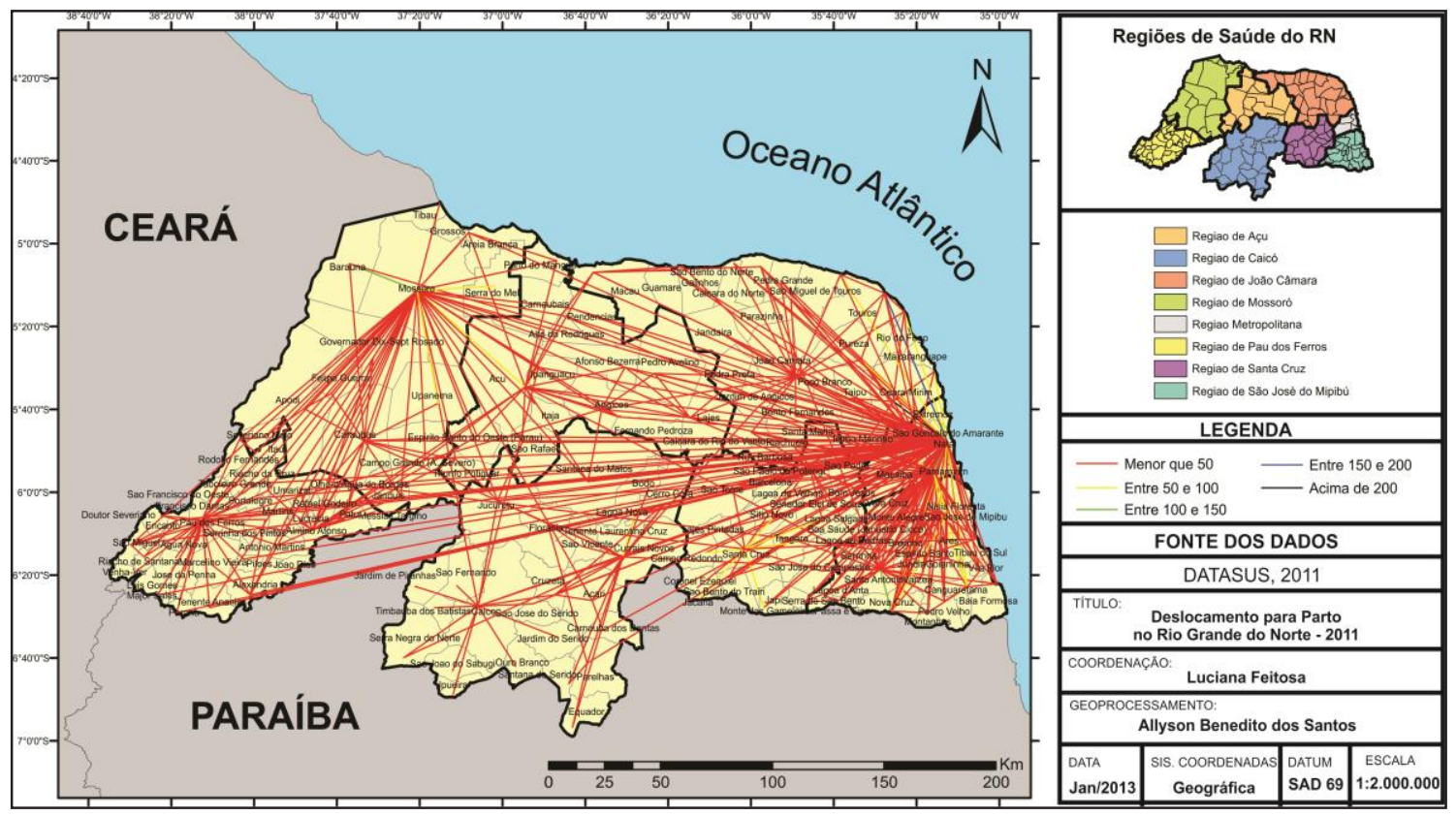

Figura 3 - Deslocamento para Parto 
A PPI praticamente não estabelece pactuação para este tipo de assistência, pois se acredita que os municípios sejam capazes de ofertar a assistência pré-natal e o serviço de parto nas maternidades municipais. Todavia, quando sobrepomos a pactuação com o fluxo real da população vemos que majoritariamente o serviço dá-se no município de Natal, que presta atendimento inclusive para a população da Região de Saúde de Pau dos Ferros. Fora a capital, outro município que se destaca no atendimento é Mossoró, que possui um raio de abrangência para a Região de Saúde de Pau dos Ferros e Região de Saúde de Assu.

Ora, imaginar um deslocamento de mais de $500 \mathrm{~km}$ no território, que é a distância entre Natal e os municípios da Região de Pau dos Ferros, para a prestação de um serviço que deve ser, majoritariamente, realizado na esfera municipal ou encaminhado para o município mais próximo, vai contra os princípios organizativos do Sistema Único de Saúde. Se as regiões foram instituídas para garantir resolutividade às demandas dos municípios que estão sob suas jurisdições administrativas e, para isso, devem ofertar os serviços essenciais à população de referência, é impraticável que um único município concentre boa parte da prestação de serviços no estado.

Sendo assim, concluímos que embora a diretriz da regionalização tenha tentado organizar a assistência à saúde em seus diversos componentes, a distribuição regional do aparato infraestrutural da saúde no estado do Rio Grande do Norte é desigual, conseqüentemente o exercício das atividades cotidianas desenvolvidas no âmbito do SUS é afetado por tal circunstância. Logo, há um descompasso entre a política de regionalização estabelecida pela Secretaria Estadual de Saúde do Rio Grande do Norte e a realidade da assistência à saúde que se dá nos lugares.

Para ser efetiva, a federalização do sistema de saúde demanda o preenchimento da forma (entes federativos) com os conteúdos do território usado. Estes fatores interferem diretamente na forma como as políticas de saúde se territorializam, por isso o processo de regionalização não pode, obrigatoriamente, obedecer à delimitação e hierarquização de áreas no território, mas, sim, atuar em acordos políticos embasados por informações coerentes com a dinâmica dos lugares.

\section{REFERÊNCIA BIBLIOGRÁFICA}

BRASIL. Ministério da Saúde. Decreto n 7. 508, de 28 de junho de 2011.

BRASIL. Ministério da Saúde. Departamento de Apoio à Descentralização. Regionalização Solidária e Cooperativa: orientações para sua implementação no SUS. Brasília: Ministério da Saúde, 2006.

GUIMARÃES, Raul Borges. A Regionalização da Saúde no Brasil: da escala do corpo à escala da nação. São Paulo: USP, 2009. Tese de Livre Docência - Faculdade de Saúde Pública, Universidade de São Paulo, 2009.

MENDES, Eugênio Vilaça. As Redes de Atenção à Saúde. Brasília: Organização Pan-Americana da Saúde, 2011.

RIO GRANDE DO NORTE. Plano Estadual de Saúde do Rio Grande do Norte: gestão 2008/2010 - vigência 2010/2011. SESAP/RN, 2008.

RIO GRANDE DO NORTE. Projeto de Regionalização do SAMU 192 no estado do Rio Grande do Norte. SESAP/RN, 2009.

SANTOS, Milton. Desafio do Ordenamento Territorial: “o pensamento”. São Paulo, 1994.

SANTOS, Milton. A Natureza do Espaço: técnica e tempo, razão e emoção. São Paulo: Edusp, 2008.

Trabalho enviado em novembro de 2012

Trabalho aceito em janeiro de 2013 